\title{
The existence of solutions of periodic BVP for second order impulsive differential equations
}

Jingli Xie ${ }^{1,2}$ and Zhiguo Luo ${ }^{2 *}$

"Correspondence:

luozg@hunnu.edu.cn

${ }^{2}$ Department of Mathematics, Hunan Normal University,

Changsha, Hunan 410081, P.R. China

Full list of author information is

available at the end of the article

\begin{abstract}
In this paper, we study the existence of solutions of periodic boundary value problems for impulsive differential equations depending on a parameter $\lambda$. By employing an existing critical point theorem, we find the range of the control parameter in which the boundary value problem admits at least one non-zero weak solution. An example illustrates our results.
\end{abstract}

MSC: 34B15; 34B18; 34B37; 58E30

Keywords: critical point theorem; impulsive equations; boundary value problem

\section{Introduction}

The well-known impulsive differential equations serve as basic models to study the dynamics of processes that are subject to sudden changes in their states, which are often investigated in various fields of science and technology [1-3]. For example, in the motion of spacecraft, one has to consider instantaneous impulses at a position with jump discontinuities in velocity, but no change in the position [4-7]. This motivates us to consider the following particular periodic boundary value problems:

$$
\left\{\begin{array}{l}
-u^{\prime \prime}(t)+u(t)=\lambda f(t, u(t)), \quad t \neq t_{j}, t \in[0,1] \\
\Delta u^{\prime}\left(t_{j}\right)=I_{j}\left(u\left(t_{j}\right)\right), \quad j=1,2, \ldots, m, \\
u(0)-u(1)=u^{\prime}(0)-u^{\prime}(1)=0
\end{array}\right.
$$

where $f:[0,1] \times \mathbb{R} \rightarrow \mathbb{R}, I_{j} \in C(\mathbb{R}, \mathbb{R}), 0=t_{0}<t_{1}<t_{2}<\cdots<t_{m}<t_{m+1}=1, \lambda$ is a positive real parameter and the operator $\Delta$ is defined as $\Delta u^{\prime}\left(t_{j}\right)=u^{\prime}\left(t_{j}^{+}\right)-u^{\prime}\left(t_{j}^{-}\right)$, where $u^{\prime}\left(t_{j}^{+}\right)\left(u^{\prime}\left(t_{j}^{-}\right)\right)$ denotes the right-hand (left-hand) limit of $u^{\prime}$ at $t_{j}$.

In the literature, some classical tools have been used to study impulsive differential equations. These classical techniques include some fixed point theorems, the lower and upper solutions and the coincidence degree theory [8-10]. Moreover, in the last few years, some researchers have gradually paid more attention to applying variational methods to deal with the existence of solutions for impulsive differential equation boundary value problems [11-16]. The same tool has also already been used for a Neumann nonlinear differential problem in [17] (see also [18, 19] and [20] for two-point and mixed problems). In this paper, we use critical point theory and variational methods to establish the existence of at least one weak solution for problem (1.1).

C 2013 Xie and Luo; licensee Springer. This is an Open Access article distributed under the terms of the Creative Commons Attribution License (http://creativecommons.org/licenses/by/2.0), which permits unrestricted use, distribution, and reproduction in any medium, provided the original work is properly cited. 
The rest of this paper is organized as follows. In Section 2 we present several definitions and main tools. In Section 3, under suitable hypotheses, we prove that problem (1.1) possesses at least one non-zero weak solution when $\lambda$ lies in an exactly determined open interval. Finally, an example is provided to verify our results.

\section{Preliminaries}

In the following, we first introduce some notations. Take $X:=\left\{u(t) \mid u(t) \in W^{1,2}([0,1])\right.$, $u(0)=u(1)\}$, in which we consider the inner product

$$
(u, v)=\int_{0}^{1} u^{\prime}(t) v^{\prime}(t) d t+\int_{0}^{1} u(t) v(t) d t
$$

and the norm

$$
\|u\|_{X}=\left(\int_{0}^{1}\left|u^{\prime}(t)\right|^{2} d t+\int_{0}^{1}|u(t)|^{2} d t\right)^{\frac{1}{2}}
$$

Note that this norm is equivalent to the usual norm

$$
\|u\|=\left(\int_{0}^{1}\left|u^{\prime}(t)\right|^{2} d t\right)^{\frac{1}{2}}
$$

Definition 2.1 $f:[0,1] \times R \rightarrow R$ is an $L^{1}$-Carathéodory function if:

(i) $t \mapsto f(t, u)$ is measurable for every $u \in \mathbb{R}$;

(ii) $u \mapsto f(t, u)$ is continuous for almost every $t \in[0,1]$;

(iii) for every $s>0$, there exists a function $l_{s} \in L^{1}([0,1])$ such that

$$
\sup _{|u| \leq s}|f(t, u)| \leq l_{s}(t) \quad \text { for a.e. } t \in[0,1]
$$

Definition 2.2 The function $u:[0,1] \rightarrow R$ is called a weak solution of problem (1.1) if $u \in X$ and

$$
\int_{0}^{1} u^{\prime}(t) v^{\prime}(t) d t+\int_{0}^{1} u(t) v(t) d t+\sum_{j=1}^{m} I_{j}\left(u\left(t_{j}\right)\right) v\left(t_{j}\right)=\lambda \int_{0}^{1} f(t, u(t)) v(t) d t
$$

for all $v \in X$.

Note that if $f$ is continuous, each weak solution is a classical solution of problem (1.1), i.e., $u \in C^{2}\left(t_{j-1}, t_{j}\right)$, satisfies the equation of (1.1) a.e. on $t \in[0,1]$, the limits $u^{\prime}\left(t_{j}^{+}\right), u^{\prime}\left(t_{j}^{-}\right)$, $j=1,2, \ldots, m$, exist and $\Delta u^{\prime}\left(t_{j}\right)=I_{j}\left(u\left(t_{j}\right)\right)$ holds.

We recall the following inequality which will be used later.

Lemma 2.3 If $u \in X$, then

$$
\|u\|_{\infty} \leq \sqrt{2}\|u\|_{X}
$$

where $\|u\|_{\infty}=\max _{t \in[0,1]}|u(t)|$. 
Proof The proof follows easily from the mean value theorem and the Hölder inequality, so we omit it here.

Next we define a functional $\varphi_{\lambda}$ as

$$
\varphi_{\lambda}(u)=\Phi(u)-\lambda \Psi(u), \quad u \in X
$$

where

$$
\Phi(u)=\frac{1}{2}\|u\|_{X}^{2}+\sum_{j=1}^{m} \int_{0}^{u\left(t_{j}\right)} I_{j}(s) d s
$$

and

$$
\Psi(u)=\int_{0}^{1} F(t, u(t)) d t,
$$

with

$$
F(t, u)=\int_{0}^{u(t)} f(t, s) d s .
$$

Note that $\varphi_{\lambda}$ is Fréchet differentiable at any $u \in X$ and for any $v \in X$, we have

$$
\begin{aligned}
\varphi_{\lambda}^{\prime}(u)(v)= & \int_{0}^{1}\left(u^{\prime}(t) v^{\prime}(t)+u(t) v(t)\right) d t+\sum_{j=1}^{m} I_{j}\left(u\left(t_{j}\right)\right) v\left(t_{j}\right) \\
& -\lambda \int_{0}^{1} f(t, u(t)) v(t) d t .
\end{aligned}
$$

Obviously, $\varphi_{\lambda}^{\prime}$ is continuous and a critical point of $\varphi_{\lambda}$, by (2.5), gives a weak solution of problem (1.1).

For all $r_{1}, r_{2} \in \mathbb{R}$, with $r_{1}<r_{2}$, we define

$$
\begin{aligned}
& \beta\left(r_{1}, r_{2}\right)=\inf _{v \in \Phi^{-1}\left(\left(r_{1}, r_{2}\right)\right)} \frac{\sup _{u \in \Phi^{-1}\left(\left(r_{1}, r_{2}\right)\right)} \Psi(u)-\Psi(v)}{r_{2}-\Phi(v)}, \\
& \alpha\left(r_{1}, r_{2}\right)=\sup _{v \in \Phi^{-1}\left(\left(r_{1}, r_{2}\right)\right)} \frac{\Psi(v)-\sup _{u \in \Phi^{-1}\left(\left(-\infty, r_{1}\right)\right)} \Psi(u)}{\Phi(v)-r_{1}} .
\end{aligned}
$$

Note that for all $r_{1}, r_{2} \in \mathbb{R}$, with $r_{1}<r_{2}$, we have $\beta\left(r_{1}, r_{2}\right) \geq 0, \alpha\left(r_{1}, r_{2}\right) \geq 0$.

To prove our main results, we need the following critical point theorem.

Theorem 2.4 [21, Theorem 5.1] Let $X$ be a reflexive real Banach space. Let $\Phi: X \rightarrow R$ be a sequentially weakly lower semicontinuous, coercive and continuously Gâteaux differentiable functional whose Gâteaux derivative admits a continuous inverse on $X^{*}$; let $\Psi: X \rightarrow R$ be a continuously Gâteaux differentiable functional whose Gáteaux derivative is compact. Put $\varphi_{\lambda}=\Phi-\lambda \Psi$ and assume that there are $r_{1}, r_{2} \in \mathbb{R}$, with $r_{1}<r_{2}$, such that

$$
\beta\left(r_{1}, r_{2}\right)<\alpha\left(r_{1}, r_{2}\right)
$$


where $\beta$ and $\alpha$ are given by (2.6) and (2.7). Then, for each $\lambda \in\left(1 / \alpha\left(r_{1}, r_{2}\right), 1 / \beta\left(r_{1}, r_{2}\right)\right)$, there is $u_{0, \lambda} \in \Phi^{-1}\left(\left(r_{1}, r_{2}\right)\right)$ such that $\varphi_{\lambda}\left(u_{0, \lambda}\right) \leq \varphi_{\lambda}(u)$ for all $u \in \Phi^{-1}\left(\left(r_{1}, r_{2}\right)\right)$ and $\varphi_{\lambda}^{\prime}\left(u_{0, \lambda}\right)=0$.

For the sake of convenience, we list the following conditions.

(H1) $f$ is an $L^{1}$-Carathéodory function.

(H2) $0 \leq \int_{0}^{u} I_{j}(s) d s<\frac{|u|^{2}}{2 m}, u \in \mathbb{R}, j=1,2, \ldots, m$.

\section{Main results}

In this section, we establish existence results for the periodic boundary value problem (1.1).

Given three nonnegative constants $c_{1}, c_{2}$, $d$, with $c_{1}<\sqrt{2} d<2 d<c_{2}$, put

$$
a\left(c_{2}, d\right)=4 \frac{\int_{0}^{1} \max _{|u(t)| \leq c_{2}} F(t, u(t)) d t-\int_{0}^{1} F(t, d) d t}{c_{2}^{2}-4 d^{2}}
$$

and

$$
b\left(c_{1}, d\right)=4 \frac{\int_{0}^{1} F(t, d) d t-\int_{0}^{1} \max _{|u(t)| \leq c_{1}} F(t, u(t)) d t}{4 d^{2}-c_{1}^{2}} .
$$

Theorem 3.1 Assume that (H1), (H2) are satisfied and there exist three nonnegative constants $c_{1}, c_{2}, d$, with $c_{1}<\sqrt{2} d<2 d<c_{2}$, such that

$$
a\left(c_{2}, d\right)<b\left(c_{1}, d\right)
$$

Then, for each $\lambda \in\left(1 / b\left(c_{1}, d\right), 1 / a\left(c_{2}, d\right)\right)$, problem (1.1) admits at least one weak solution $u(t), t \in[0,1]$, such that $\sqrt{6} c_{1} / 6<\|u\|_{X}<\sqrt{2} c_{2} / 2$.

Proof By (2.3) and (2.4), we have that $\Phi$ is a nonnegative Gâteaux differentiable, coercive and sequentially weakly lower semicontinuous functional whose Gâteaux derivative admits a continuous inverse on $X^{*}$, and $\Psi$ is a continuously Gâteaux differentiable functional whose Gâteaux is compact. Let

$$
r_{1}=\frac{c_{1}^{2}}{4}, \quad r_{2}=\frac{c_{2}^{2}}{4}, \quad u_{0}(t)=d, \quad \text { for } t \in[0,1] .
$$

By condition (H2), we have

$$
\begin{aligned}
\Phi\left(u_{0}\right) & =\frac{1}{2}\left\|u_{0}\right\|_{X}^{2}+\sum_{j=1}^{m} \int_{0}^{u_{0}\left(t_{j}\right)} I_{j}(s) d s \\
& =\frac{1}{2} d^{2}+\sum_{j=1}^{m} \int_{0}^{d} I_{j}(s) d s \\
& \leq d^{2}
\end{aligned}
$$

and

$$
\Phi\left(u_{0}\right) \geq \frac{d^{2}}{2}
$$


Combining $c_{1}<\sqrt{2} d<2 d<c_{2}$, (3.4), (3.5) and (3.6), we have

$$
r_{1}<\Phi\left(u_{0}\right)<r_{2} .
$$

Clearly, we have $\Psi\left(u_{0}\right)=\int_{0}^{1} F\left(t, u_{0}(t)\right) d t=\int_{0}^{1} F(t, d) d t$. From Lemma 2.3, the estimate $\Phi(u)<r_{2}, u \in X$, implies that

$$
|u(t)|^{2} \leq 2\|u\|_{X}^{2} \leq 2 \times 2 \Phi(u)<4 r_{2}=c_{2}^{2}, \quad t \in[0,1],
$$

and

$$
\int_{0}^{1} F(t, u(t)) d t \leq \int_{0}^{1} \max _{|u(t)| \leq c_{2}} F(t, u) d t
$$

Therefore

$$
\sup _{u \in \Phi^{-1}\left(\left(r_{1}, r_{2}\right)\right)} \Psi(u) \leq \sup _{u \in \Phi^{-1}\left(\left(-\infty, r_{2}\right)\right)} \Psi(u) \leq \int_{0}^{1} \max _{|u(t)| \leq c_{2}} F(t, u(t)) d t .
$$

For $u \in X$ with $\Phi(u)<r_{1}$, one can similarly obtain

$$
\sup _{u \in \Phi^{-1}\left(\left(-\infty, r_{1}\right)\right)} \Psi(u) \leq \int_{0}^{1} \max _{|u(t)| \leq c_{1}} F(t, u(t)) d t
$$

Therefore, we have

$$
\begin{aligned}
\beta\left(r_{1}, r_{2}\right) & \leq \frac{\sup _{u \in \Phi^{-1}\left(\left(-\infty, r_{2}\right)\right)} \Psi(u)-\Psi\left(u_{0}\right)}{r_{2}-\Phi\left(u_{0}\right)} \\
& \leq 4 \frac{\int_{0}^{1} \max _{|u(t)| \leq c_{2}} F(t, u(t)) d t-\int_{0}^{1} F(t, d) d t}{c_{2}^{2}-4 d^{2}} \\
& =a\left(c_{2}, d\right) .
\end{aligned}
$$

On the other hand, we have

$$
\begin{aligned}
\alpha\left(r_{1}, r_{2}\right) & \geq \frac{\Psi\left(u_{0}\right)-\sup _{u \in \Phi^{-1}\left(\left(-\infty, r_{1}\right)\right)} \Psi(u)}{\Phi\left(u_{0}\right)-r_{1}} \\
& \geq 4 \frac{\int_{0}^{1} F(t, d) d t-\int_{0}^{1} \max _{|u(t)| \leq c_{1}} F(t, u(t)) d t}{4 d^{2}-c_{1}^{2}} \\
& =b\left(c_{1}, d\right) .
\end{aligned}
$$

So, by (3.3), we induce

$$
\beta\left(r_{1}, r_{2}\right)<\alpha\left(r_{1}, r_{2}\right) .
$$

Therefore, by Theorem 2.4, for each $\lambda \in\left(1 / b\left(c_{1}, d\right), 1 / a\left(c_{2}, d\right)\right)$, we have that $\Phi-\lambda \Psi$ admits at least one critical point $u$ such that $r_{1}<\Phi(u)<r_{2}$. Combining (2.3), we get

$$
\frac{c_{1}^{2}}{4}<\frac{1}{2}\|u\|_{X}^{2}+\sum_{j=1}^{m} \int_{0}^{u\left(t_{j}\right)} I_{j}(s) d s \leq \frac{3}{2}\|u\|_{X}^{2},
$$


and

$$
\frac{1}{2}\|u\|_{X}^{2} \leq \frac{1}{2}\|u\|_{X}^{2}+\sum_{j=1}^{m} \int_{0}^{u\left(t_{j}\right)} I_{j}(s) d s<\frac{c_{2}^{2}}{4} .
$$

So, problem (1.1) admits at least one weak solution $u(t), t \in[0,1]$, such that $\frac{\sqrt{6}}{6} c_{1}<\|u\|_{X}<$ $\frac{\sqrt{2}}{2} c_{2}$.

Theorem 3.2 Assume that (H1), (H2) hold and there exist two positive constants $c, d$, with $2 d<c$, such that

$$
\left(\int_{0}^{1} \max _{|u(t)| \leq c} F(t, u(t)) d t\right) / c^{2}<\left(\int_{0}^{1} F(t, d) d t\right) / 4 d^{2} .
$$

Then, for each $\lambda \in\left(d^{2} / \int_{0}^{1} F(t, d) d t, c^{2} / 4 \int_{0}^{1} \max _{|u(t)| \leq c} F(t, u(t)) d t\right)$, problem (1.1) admits at least one nontrivial weak solution $u(t), t \in[0,1]$, such that $\|u\|_{X}<\frac{\sqrt{2}}{2} c$.

Proof Let $c_{1}=0$ and $c_{2}=c$, then by (3.1) and (3.2) we get

$$
\begin{aligned}
a\left(c_{2}, d\right) & =4 \frac{\int_{0}^{1} \max _{|u(t)| \leq c_{2}} F(t, u(t)) d t-\int_{0}^{1} F(t, d)}{c_{2}^{2}-4 d^{2}} \\
& \leq 4 \frac{\int_{0}^{1} \max _{|u(t)| \leq c} F(t, u(t)) d t-\frac{4 d^{2}}{c^{2}} \int_{0}^{1} \max _{|u(t)| \leq c} F(t, u(t))}{c^{2}-4 d^{2}} \\
& =4 \frac{\int_{0}^{1} \max _{|u(t)| \leq c} F(t, u(t)) d t}{c^{2}}
\end{aligned}
$$

and

$$
b\left(c_{1}, d\right)=4 \frac{\int_{0}^{1} F(t, d) d t}{4 d^{2}}
$$

Therefore, owing to (3.6) we have $a(c, d)<b(0, d)$. Moreover, by Theorem 3.1, we have that for each $\lambda \in\left(d^{2} / \int_{0}^{1} F(t, d) d t, c^{2} / 4 \int_{0}^{1} \max _{|u(t)| \leq c} F(t, u(t)) d t\right)$, problem (1.1) admits at least one nontrivial weak solution $u$ such that $\|u\|_{X}<\frac{\sqrt{2}}{2} c$.

Now, when the nonlinear term of problem (1.1) is with separable variables, we have the following results. To be precise, let $\alpha \in L^{1}([0,1])$ be such that $\alpha(t) \geq 0$ a.e. $t \in[0,1], \alpha \neq 0$, and let $g: \mathbb{R} \rightarrow \mathbb{R}$ be a nonnegative continuous function. Consider the boundary value problem

$$
\left\{\begin{array}{l}
-u^{\prime \prime}(t)+u(t)=\lambda \alpha(t) g(u(t)), \quad t \neq t_{j}, t \in[0,1] \\
\Delta u^{\prime}\left(t_{j}\right)=I_{j}\left(u\left(t_{j}\right)\right), \quad j=1,2, \ldots, m, \\
u(0)-u(1)=u^{\prime}(0)-u^{\prime}(1)=0 .
\end{array}\right.
$$

Put

$$
G(u)=\int_{0}^{u(t)} g(s) d s \quad \text { for } u \in X
$$


Corollary 3.3 Assume that (H2) is satisfied and there exist three nonnegative constants $c_{1}, c_{2}, d$, with $c_{1}<\sqrt{2} d<2 d<c_{2}$, such that

$$
\frac{G\left(c_{2}\right)-G(d)}{c_{2}^{2}-4 d^{2}}<\frac{G(d)-G\left(c_{1}\right)}{4 d^{2}-c_{1}^{2}}
$$

Then, for each $\lambda \in\left(\left(4 d^{2}-c_{1}^{2}\right) / 4\|\alpha\|_{1}\left(G(d)-G\left(c_{1}\right)\right),\left(c_{2}^{2}-4 d^{2}\right) / 4\|\alpha\|_{1}\left(G\left(c_{2}\right)-G(d)\right)\right)$, where $\|\alpha\|_{1}=\int_{0}^{1}|\alpha(t)| d t$, problem (3.8) admits at least one weak solution $u(t), t \in[0,1]$, such that $\frac{\sqrt{6}}{6} c_{1}<\|u\|_{X}<\frac{\sqrt{2}}{2} c_{2}$.

Proof Let $f(t, u)=\alpha(t) g(u)$ for all $(t, u) \in[0,1] \times \mathbb{R}$. It is clear that $F(t, u)=\alpha(t) G(u)$ for all $(t, u) \in[0,1] \times X$. Moreover, $G$ is a nondecreasing function about $u$. So, we have

$$
a\left(c_{2}, d\right)=4\|\alpha\|_{1} \frac{G\left(c_{2}\right)-G(d)}{c_{2}^{2}-4 d^{2}}<4\|\alpha\|_{1} \frac{G(d)-G\left(c_{1}\right)}{4 d^{2}-c_{1}^{2}}=b\left(c_{1}, d\right) .
$$

So, for each $\lambda \in\left(\left(4 d^{2}-c_{1}^{2}\right) / 4\|\alpha\|_{1}\left(G(d)-G\left(c_{1}\right)\right),\left(c_{2}^{2}-4 d^{2}\right) / 4\|\alpha\|_{1}\left(G\left(c_{2}\right)-G(d)\right)\right)$, problem (3.8) admits at least one weak solution $u(t), t \in[0,1]$, such that $\frac{\sqrt{6}}{6} c_{1}<\|u\|_{X}<\frac{\sqrt{2}}{2} c_{2}$.

Corollary 3.4 Assume that $(\mathrm{H} 2)$ is satisfied and there exist two positive constants $c, d$, with $c>2 d$, such that

$$
\frac{G(c)}{c^{2}}<\frac{G(d)}{4 d^{2}}
$$

Then, for each $\lambda \in\left(d^{2} / G(d)\|\alpha\|_{1}, c^{2} / 4 G(c)\|\alpha\|_{1}\right)$, problem (3.8) admits at least one weak solution $u(t)$ such that $|u(t)|<c$ for all $t \in[0,1]$.

Proof Let $f(t, u)=\alpha(t) g(u)$ for all $(t, u) \in[0,1] \times \mathbb{R}$. It is clear that $F(t, u)=\alpha(t) G(u)$ for all $(t, u) \in[0,1] \times X$. Moreover, $G$ is a nondecreasing function about $u$. So, we have

$$
\begin{aligned}
& \frac{\int_{0}^{1} \max _{|u(t)| \leq c} F(t, u(t)) d t}{c^{2}}=\frac{\|\alpha\|_{1} G(c)}{c^{2}}<\frac{\|\alpha\|_{1} G(d)}{4 d^{2}}=\frac{\int_{0}^{1} F(t, d) d t}{4 d^{2}}, \\
& \frac{d^{2}}{\int_{0}^{1} F(t, d) d t}=\frac{d^{2}}{G(d)\|\alpha\|_{1}}, \quad \frac{c^{2}}{4 \int_{0}^{1} \max _{|u(t)| \leq c} F(t, u(t)) d t}=\frac{c^{2}}{4 G(c)\|\alpha\|_{1}} .
\end{aligned}
$$

Therefore, by Theorem 3.2, for each $\lambda \in\left(d^{2} / G(d)\|\alpha\|_{1}, c^{2} / 4 G(c)\|\alpha\|_{1}\right)$, problem (3.8) admits at least one weak solution $u(t)$ such that $|u(t)|<c$ for all $t \in[0,1]$.

\section{An example}

In this section, we give an example to illustrate our main results.

Example 4.1 Consider the boundary value problem

$$
\left\{\begin{array}{l}
-u^{\prime \prime}(t)+u=2 \lambda t e^{-u}, \quad t \neq t_{1}, t \in[0,1] \\
\Delta u^{\prime}\left(t_{1}\right)=\frac{1}{2} u\left(t_{1}\right), \quad t_{1}=\frac{1}{2} \\
u(0)-u(1)=u^{\prime}(0)-u^{\prime}(1)=0
\end{array}\right.
$$


Compared to problem (3.8), $\alpha(t)=2 t, g(u)=e^{-u}, I_{j}(u)=\frac{1}{2} u$. Clearly, (H2) is satisfied and $\alpha \in L^{1}([0,1])$ such that $\alpha(t) \geq 0$ a.e. $t \in[0,1], \alpha \neq \equiv$, and $g: R \rightarrow R$ is a nonnegative continuous function. Choose $d=0.5, c=2$. By simple calculations, we obtain

$$
\begin{aligned}
& \frac{G(c)}{c^{2}} \approx 0.2162<\frac{G(d)}{4 d^{2}} \approx 0.3934 \\
& \frac{d^{2}}{G(d)\|\alpha\|_{1}} \approx 0.6355, \quad \frac{c^{2}}{4 G(c)\|\alpha\|_{1}} \approx 1.1566 .
\end{aligned}
$$

Applying Corollary 3.4, when $\lambda \in(0.6355,1.1566)$, system $(4.1)$ has at least one weak solution $u$ such that $|u(t)|<2$ for all $t \in[0,1]$.

\section{Competing interests}

The authors declare that they have no competing interests.

\section{Authors' contributions}

JX drafted the manuscript and joined discussion with ZL. ZL read the draft and provided comments. Both authors read and approved the final version of the manuscript.

\section{Author details}

${ }^{1}$ College of Mathematics and Statistics, Jishou University, Jishou, Hunan 416000, P.R. China. ${ }^{2}$ Department of Mathematics, Hunan Normal University, Changsha, Hunan 410081, P.R. China.

\section{Acknowledgements}

The authors thank the referees for their valuable comments and suggestions, which greatly improved the presentation of this paper. This work is supported by Hunan Provincial Natural Science Foundation of China (No: 11 JJ3012), Major Project of Science Research Fund of Education Department in Hunan (No: 11A095).

\section{Received: 30 March 2013 Accepted: 1 August 2013 Published: 22 August 2013}

\section{References}

1. Benchohra, M, Henderson, J, Ntouyas, S: Theory of Impulsive Differential Equations and Inclusions. Contemporary Mathematics and Its Applications, vol. 2. Hindawi Publishing Corporation, New York (2006)

2. Bainov, DD, Simeonov, PS: Systems with Impulse Effect: Stability Theory and Applications. Ellis Horwood, Chichester (1989)

3. Jing, G, Lu, Q: Impulsive state feedback control of a predator-prey model. J. Comput. Appl. Math. 200, 193-207 (2007)

4. Agarwal, RP, Franco, D, Regan, DO: Singular boundary value problems for first and second order impulsive differential equations. Aequ. Math. 69, 83-96 (2005)

5. Carter, TE: Necessary and sufficient conditions for optimal impulsive rendezvous with linear equations of motion. Dyn. Control 10, 219-227 (2000)

6. Carter, TE: Optimal impulsive space trajectories based on linear equations. J. Optim. Theory Appl. 70, 277-297 (1991)

7. Prado, AFBA: Bi-impulsive control to build a satellite constellation. Nonlinear Dyn. Syst. Theory 5, 169-175 (2005)

8. Agarwal, RP, Regan, DO: A multiplicity result for second order impulsive differential equations via the Leggett Williams fixed point theorem. Appl. Math. Comput. 161, 433-439 (2005)

9. Qin, D, Li, X: Periodic solutions for ordinary differential equations with sublinear impulsive effects. J. Math. Anal. Appl. 303, 288-303 (2005)

10. Shen, J, Wang, B: Impulsive boundary value problems with nonlinear boundary conditions. Nonlinear Anal. 69 , 4055-4062 (2008)

11. Marek, G, Szymon, G: On the discrete boundary value problem for anisotropic equation. J. Math. Anal. Appl. 386, 956-965 (2012)

12. Nieto, JJ, Regan, DO: Variational approach to impulsive differential equations. Nonlinear Anal., Real World Appl. 10 , 680-690 (2009)

13. Tian, Y, Ge, W: Applications of variational methods to boundary-value problem for impulsive differential equations. Proc. Edinb. Math. Soc. 51, 509-527 (2008)

14. Zhang, H, Li, Z: Variational approach to impulsive differential equations with periodic boundary conditions. Nonlinear Anal., Real World Appl. 11, 67-78 (2010)

15. Zhang, $X$, Tang, $X$ : Subharmonic solutions for a class of non-quadratic second order Hamiltonian systems. Nonlinear Anal., Real World Appl. 13, 113-130 (2012)

16. Zhang, Z, Yuan, R: Applications of variational methods to Dirichlet boundary value problem with impulses. Nonlinear Anal., Real World Appl. 11, 155-162 (2010)

17. Bonanno, G, Pizzimenti, PF: Neumann boundary value problems with not coercive potential. Mediterr. J. Math. 33 173-191 (2010)

18. Bonanno, G, Sciammetta, A: An existence result of one non-trivial solution for two point boundary value problems. Bull. Aust. Math. Soc. 84, 288-299 (2011)

19. Aguì, GD: Existence results for a mixed boundary value problem with Sturm-Liouville equation. Adv. Pure Appl. Math. 2, 237-248 (2011) 
20. Aguì, GD: Multiplicity results for nonlinear mixed boundary value problem. Bound. Value Probl. 2012, 134 (2012)

21. Bonanno, G: A critical point theorem via the Ekeland variational principle. Nonlinear Anal. 75, 2992-3007 (2012)

doi:10.1186/1029-242X-2013-406

Cite this article as: Xie and Luo: The existence of solutions of periodic BVP for second order impulsive differential equations. Journal of Inequalities and Applications 2013 2013:406.

Submit your manuscript to a SpringerOpen ${ }^{\circ}$ journal and benefit from:

- Convenient online submission

- Rigorous peer review

- Immediate publication on acceptance

Open access: articles freely available online

- High visibility within the field

- Retaining the copyright to your article

Submit your next manuscript at $>$ springeropen.com 